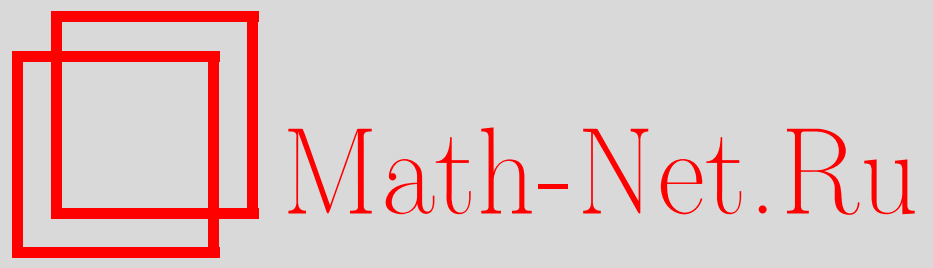

Обцероссийский математический портал

А. Балтрунас, О субэкспоненциальности одного класса случайных величин, Матем. заметки, 2001, том 69, выпуск 4, 625-628

DOI: https://doi.org/10.4213/mzm689

Использование Общероссийского математического портала Math-Net.Ru подразумевает, что вы прочитали и согласны с пользовательским соглашением http://www.mathnet.ru/rus/agreement

Параметры загрузки:

IP : 54.92 .164 .108

26 апреля 2023 г., 16:10:24 


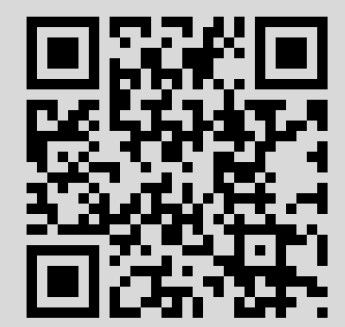




\section{О СУБЭКСПОНЕНЦИАЛЬНОСТИ ОДНОГО КЛАССА СЛУЧАЙНЫХ ВЕЛИЧИН}

\section{А. Балтрунас}

Введение. Будем говорить, что функция распределения $F$ неотрицательной случайной величины $X$ принадлежит классу $S$ субәкспоненииальных распределений, если ее хвост $\bar{F}:=1-F$ удовлетворяет соотношению

$$
\lim _{t \rightarrow \infty} \frac{\overline{F * F}(t)}{\bar{F}(t)}=2,
$$

где $*$, как обычно, означает свертку по Стилтьесу.

Известно (см. теорему 2 работы [1]), что если выполнено (1), то

$$
\lim _{t \rightarrow \infty} \frac{\bar{F}(t+y)}{\bar{F}(t)}=1, \quad y \geqslant 0 .
$$

Через $\mathbf{L}$ обозначим класс всех функций, удовлетворяющих соотношениям (2).

Функция распределения $F$ является махсорируемо меняющейся на бесконечности, если

$$
\varlimsup_{t \rightarrow \infty} \frac{\bar{F}(t)}{\bar{F}(2 t)}<\infty .
$$

Если выполнено соотношение (3), мы будем писать $F \in \mathbf{D}$. Имеем, что если $F \in \mathbf{D} \cap \mathbf{L}$, то $F \in S$.

Пусть $X$ и $Y$ - независимые неотрицательные случайные величины, имеющие функции распределения $F_{X}$ и $F_{Y}$ соответственно. Далее везде будем предполагать, что $F_{X} \in S$ и $F_{Y} \in S$. В работе [2] изучались условия, при выполнении которых функции распределения случайных величин $\max (X, Y)$ и $\min (X, Y)$ принадлежат классу $S$. В этой работе Якымив связал вопрос субэкспоненциальности случайной величины $\max (X, Y)$ с проблемой субэкспоненциальности случайной величины $X+Y$, которая уже достаточно подробно изучена (см. [3]-[6]). Более сложным оказался вопрос о принадлежности функции распределения случайной величины $\min (X, Y)$ классу $S$. Если через $V$ мы обозначим функцию распределения случайной величины $\min (X, Y)$, то в [2] доказан такой результат: если $F_{X} \in \mathbf{D} \cap S$ и $F_{Y} \in \mathbf{D} \cap S$, то $V \in S$. Цель настоящей заметки - обобщить этот результат. А именно, для достаточно шшрокого класса субэкспоненциальных распределений мы дадим достаточные условия субэкспоненциальности функции распределения $V$.

Основные результаты. Мы будем говорить, что неотрицательная неубывающая функция $g$, определенная на $[0, \infty)$, является ограниченно полуаддитивной, если $g(0)=0$ и

$$
\varlimsup_{x \rightarrow \infty}\left(g(x)-\min _{0 \leqslant y \leqslant x / 2}(g(y)+g(x-y))\right)<\infty .
$$

(C) А. БАлТРУНАС 
Определим

$$
\begin{aligned}
& R_{X}(t)=-\ln \bar{F}_{X}(t), \quad t \geqslant 0 \\
& R_{Y}(t)=-\ln \bar{F}_{Y}(t), \quad t \geqslant 0
\end{aligned}
$$

Наш основной результат представляет следующая теорема.

Теорема 1. Допустим, что $R_{X}$ и $R_{Y}$ являются ограниченно полуаддитивными функчиями. Если $F_{X} \in S$ и $F_{Y} \in S$, mo $V \in S$.

ДокАЗАТЕльство. Поскольку $F_{X} \in S$, существует функция $a_{1}(t) \rightarrow \infty$ при $t \rightarrow \infty$ такая, что

$$
\int_{a_{1}(t)}^{t} \bar{F}_{X}(t-u) d F_{X}(u)=o\left(\bar{F}_{X}(t)\right)
$$

при $t \rightarrow \infty$.

Аналогично, из $F_{Y} \in S$ следует, что для какой-то функции $a_{2}(t) \rightarrow \infty$ имеем

$$
\int_{a_{2}(t)}^{t} \bar{F}_{Y}(t-u) d F_{Y}(u)=o\left(\bar{F}_{Y}(t)\right)
$$

при $t \rightarrow \infty$.

Пусть $a(t)=\max \left(a_{1}(t), a_{2}(t)\right)$. Имеем

$$
\overline{V * V}(t)=\bar{V}(t)+\int_{0}^{t} \bar{V}(t-u) d V(u) .
$$

Поскольку $\bar{V}(t)=\mathrm{P}(\min (X, Y)>t)=\bar{F}_{X}(t) \cdot \bar{F}_{Y}(t)$, мы получаем

$$
\begin{aligned}
\int_{a(t)}^{t} \bar{V}(t-u) d V(u)= & \int_{a(t)}^{t} \bar{F}_{X}(t-u) \bar{F}_{X}(u) \bar{F}_{Y}(t-u) d F_{Y}(u) \\
& +\int_{a(t)}^{t} \bar{F}_{X}(t-u) \bar{F}_{Y}(t-u) \bar{F}_{Y}(u) d F_{X}(u) \\
= & \mathrm{I}+\mathrm{II} .
\end{aligned}
$$

Учитьвая тот факт, что $R_{X}$ является ограниченно полуаддитивной функцией, получаем, что для всех $u$ таких, что $0 \leqslant u \leqslant t / 2$, имеет место оценка

$$
\frac{\bar{F}_{X}(t-u) \bar{F}_{X}(u)}{\bar{F}_{X}(t)} \leqslant C<\infty .
$$

Тогда мы имеем

$$
\mathrm{I}=O(1) \bar{F}_{X}(t) \int_{a(t)}^{t} \bar{F}_{Y}(t-u) d F_{Y}(u)=\bar{F}_{X}(t) o\left(\bar{F}_{Y}(t)\right)=o(\bar{V}(t))
$$

при $t \rightarrow \infty$. Аналогично, из ограниченной полуаддитивности функции $R_{Y}$ вытекает, что II $=$ $o(\bar{V}(t))$ при $t \rightarrow \infty$.

Отсюда

$$
\int_{a(t)}^{t} \bar{V}(t-u) d V(u)=o(\bar{V}(t))
$$

при $t \rightarrow \infty$.

Теорема доказана.

Следующая лемма дает достаточное условие ограниченной полуадлитивности функции $g$. 
Лемма 2. Допустим, что $F \in \mathbf{D}$. Тогда функция $R=-\ln \bar{F}$ является ограниченно полуаддитивной.

ДоКАЗАТЕЛЬСТво. Поскольку $F \in \mathbf{D}$, имеем

$$
A:=\varlimsup_{t \rightarrow \infty} \sup _{0 \leqslant y \leqslant t / 2}(R(t)-R(t-y)) \leqslant \varlimsup_{t \rightarrow \infty}\left(R(t)-R\left(\frac{t}{2}\right)\right)<\infty .
$$

Тогда

$$
\begin{aligned}
H_{R}(t) & :=R(t)-\min _{0 \leqslant y \leqslant t / 2}(R(y)+R(t-y)) \leqslant R(t)-\min _{0 \leqslant y \leqslant t / 2} R(t-y) \\
& \leqslant \sup _{0 \leqslant y \leqslant t / 2}(R(t)-R(t-y)) \leqslant A<\infty,
\end{aligned}
$$

что и требовалось доказать.

Лемма доказана.

Будем говорить, что неотрицательная неубьвающая функция $g$, определенная на $[0, \infty)$, является асимптотически полуаддитивной, если $g(0)=0$ и

$$
\lim _{x \rightarrow \infty}\left(g(x)-\min _{0 \leqslant y \leqslant x / 2}(g(y)+g(x-y))\right)=0 .
$$

Как нетрудно убедиться, любая асимптотически полуаддитивная функция является ограниченно полуаддитивной.

Следующая лемма дает достаточное условие, при выполнении которого функция $g$ будет асимптотически полуаддитивной.

ЛЕмма 3. Допустим, что функция $g$ имеет представление

$$
g(t)=\int_{0}^{t} q(u) d u
$$

где $q$ - неотрицательная невозрастающая функиия. Тогда g является асимптотически полуаддитивной функиией.

ДоКАЗАТЕЛЬСТво. Имеем

$$
H_{g}(t):=g(t)-\min _{0 \leqslant y \leqslant t / 2}(g(y)+g(t-y))=-\min _{0 \leqslant y \leqslant t / 2}\left(\int_{0}^{y} q(u) d u-\int_{t-y}^{t} q(u) d u\right) .
$$

Поскольку $q$ является неотрицательной невозрастающей функцией, то для любого $y$ такого, что $0 \leqslant y \leqslant t / 2$, выполнено неравенство

Тогда

$$
\int_{0}^{y} q(u) d u \geqslant \int_{t-y}^{t} q(u) d u
$$

С другой стороны, при $y=0$ мы получаем

$$
\lim _{t \rightarrow \infty} H_{g}(t) \leqslant 0
$$

$$
H_{g}(t) \geqslant g(t)-g(t)=0 .
$$

Отсюда вытекает, что $\lim _{t \rightarrow \infty} H_{g}(t)=0$.

Лемма доказана.

Допустим, что функции $R_{X}$ и $R_{Y}$, определенные соотношениями (5), (6), имеют следующие представления:

$$
R_{X}(t)=\int_{0}^{t} q_{X}(u) d u, \quad R_{Y}(t)=\int_{0}^{t} q_{Y}(u) d u, \quad t \geqslant 0
$$

где $q_{X}$ и $q_{Y}$ - неотрицательные функции.

Из теоремы 1 и леммы 3 вытекает следующее следствие.

СЛЕДСтвИЕ 4. Допустим, что $q_{X} u q_{Y}$ монотонно стремятся $\kappa 0$. Eсли $F_{X} \in S u$ $F_{Y} \in S$, mo $V \in S$.

ЗАмечание. Рогозин в работе [7] представил теорему 1, в которой утверждается, что если $F \in S$, то $R=-\ln \bar{F}$ является асимптотически полуаддитивной функцией. 


\section{СПИСОК ЦИТИРОВАННОЙ ЛИТЕРАТУРЫ}

1. Чистяков В. П. // Теор. вероятн. и еепримен. 1964. Т. 9. № 4. С. 710-718. 2. Якымив А. Л . // Матем. заметки. 1997. Т. 62. №1. C. 138-144. 3. Cline D. B. H. // Probab. Theory Related Fields. 1986. V. 72. P. 529-557. 4. Cline D. B. H. // J. Austral. Math. Soc. Ser. A. 1987. V. 43. P. 347-365. 5. Embrechts P., Goldie C. M. // J. Austral. Math. Soc. Ser. A. 1980. V. 29. P. 243-256. 6. Leslie J. // J. Appl. Probab. 1989. V. 26. P. 58-66. 7. Рогозин Б. А. // Третья Всероссийская школа-коллоквиум по стохастическим методам (Туапсе, 17-24 сентября 1996 г.). Тезисы докладов (сост. Ю. В. Прохоров и др.). М.: ТВП, 1996. С. 141-142.

Институт математики и информатики, г. Вильнюс 